\title{
Awareness, requirements and barriers to use of Assistive Technology designed to enable independence of people suffering from Dementia (ATD)
}

\author{
Eleanor van den Heuvel*, Felicity Jowitt and Anne McIntyre \\ Brunel University, Uxbridge, Middlesex, UK
}

\begin{abstract}
This study investigated the awareness and use of assistive technology for people with dementia. The aim of the study was to explore barriers to the uptake of, and the unmet needs for, assistive technology for dementia (ATD).

The work was carried out with family carers of people with dementia because carers are often the purchasers, end users and main beneficiaries of this type of technology.

Focus groups were used to explore carers' views on ATD use, and the problems that might be addressed by ATD, in order to develop a questionnaire for dementia carers. The questionnaire was administered at Alzheimer's Society carer support groups in South Bucks and in Hillingdon Borough (UK).

None of the 16 focus group participants or the 42 respondents to the questionnaire had any personal experience of ATD. The dementia carers reported a number of concerns, problems and unmet needs that could be addressed by existing, commercially available ATD products.

Some needs for new technology development were identified in the study, but the lack of awareness of ATD appears to be the most important barrier to technology adoption.
\end{abstract}

Keywords: Alzheimer's disease, assistive technology, dementia, awareness

\section{Introduction}

Dementia is a term used to describe a collection of symptoms caused by progressive and irreversible loss of mental faculties. Prevalence of dementia is predicted to more than double between 2000 and 2050 and the proportion of working age people (ie potential carers), to people with dementia is predicted to fall to less than one third of present levels [1]. It will therefore be increasingly important to find ways to ease the care burden of dementia carers and increase the potential for people with dementia to manage in their own homes.

* Address for correspondence: Eleanor van den Heuvel, Brunel Institute for Bioengineering, Brunel University, Uxbridge, Middlesex, UB8 2PH,UK. Tel.: +44 (0)1895 266921; E-mail: Eleanor.van.den. heuvel@brunel.ac.uk.
Most dementia carers in the UK are either relatives or friends of the sufferer and the care they provide (often more than 50 hours a week), is unpaid. Although most of the 5 million family carers in Britain are of working age [2], the majority of people caring for Alzheimer's disease sufferers are spouses of the dementia patient [3], and therefore they tend to be older than the general population of carers who include parents of children with disabilities, families of accident victims etc. The burden of care can be particularly heavy for those caring for a person with dementia. An American survey of 1500 carers in 1996 found that looking after someone with dementia took more hours per week and had a larger impact on the physical and mental health of the carer than other types of care giving [4]. Caring for a person with dementia has even 
been found to increase mortality in caregivers [5]. In 2006 Georges et al. [6] analysed questionnaire responses for 1181 dementia carers across five different European countries (Germany, Poland, France, Scotland and Spain). Most respondents were female (67\%) and most $(65 \%)$ were over 55 years old. The largest group of carers were looking after a spouse/partner and the next largest group were caring for a parent, with $65 \%$ living with the person they looked after.

The King's fund definition of assistive technology is "any product or service designed to enable independence for disabled and older people." [7]. This definition covers technology used by the disabled person themselves and also any technology used by their carers, which enables "independence". This is taken to mean improving both safety and functional abilities for the disabled person.

A large American study of older people using basic Assistive technology (AT) such as mobility aids found that older people who use AT need significantly fewer hours of personal assistance than people with similar levels of impairment who do not use AT [8]. The ENABLE project 2001-2004 developed and tested six different Assistive Technologies designed to support people with memory loss and dementia symptoms (ATD). This study found that the majority of carers reported positive benefits from using the devices and more than one-third reported that the emotional burden of worry was reduced by ATD use [9].

In addition to the stand alone devices, there has also been a great deal of interest in "Telecare". Telecare technology is generally a package of sensors and technologies, such as community alarm services, sensors that monitor the home environment to detect problems, reminding devices, wander alarms and environmental controls, which are monitored and supported by a central call centre. These integrated telecare systems have been reported on by several authors $[10,11]$. Much of the interesting work in the ATD field is still in the development stages, such as the use of computer vision to monitor vulnerable people [12], devices to stimulate cognition [13] and artificial intelligence to create cognitive orthoses for people with deteriorating abilities [14-16].

The aim of this study was to investigate the awareness and use of assistive technology designed specifically for people with dementia (ATD). The type of ATD used and barriers to up-take were also examined. This study only considered Assistive Technology that could ameliorate the problems caused by the loss of cognitive abilities caused by dementia, so although many people with dementia also use mobility aids for example, this type of technology was not a part of the study. The focus of this study is ATD, to discover the extent of carers' awareness of the technology and to find out whether the problems the carers highlighted were those that could be ameliorated by ATD technology that is currently available.

\section{Methods}

\subsection{Study context}

This study took place during 2007-2008 within carer support groups in two local areas; the South Buckinghamshire Alzheimer's Society and the Hillingdon and Harrow Alzheimer's Society. The areas covered by these two local branches are in the South East of the UK, close to London (South Buckinghamshire) and a borough in the west London suburbs (Harrow and Hillingdon). The population in these areas tends to be economically privileged in comparison to other areas in the UK, so one might expect that they would have better opportunities for purchasing ATD.

The South Buckinghamshire society, covering Amersham, High Wycombe and Beaconsfield areas, runs active carer support groups which meet on a monthly basis. Harrow and Hillingdon run two "Templeton Café" groups which carers and dementia sufferers can attend together; these groups meet weekly.

\subsection{Study design}

This study was designed in two parts: 1) A qualitative focus group methodology was used to aid the development of a questionnaire; 2) The questionnaire was then used to survey dementia carers in order to understand their experiences relating to ATD.

The focus group method was chosen as a means of discovering the topics that were important and relevant for inclusion in the questionnaire to enable an exploration of carers views on ATD use, and the problems that might be addressed by ATD. This method was selected because it allows a lot of data to be gathered in a short time and interaction between participants generates richer data than would be obtained from individual interviews [17]. The focus group is also a well established means of obtaining data in an under researched area [18] enabling questionnaires to be developed from topics of concern to users. The two focus groups took place at one of the Amersham-based carer 
support groups. The project was introduced to the full carer's group $(\sim 25$ people) one month before the focus groups were to take place. The following month two focus group sessions of approximately 30 minutes each were held consecutively. An interview guide, covering the use/non-use and awareness of ATD, technology that could be developed and problems that need to be solved, was used to guide both focus group discussions. Carers were also asked about AT for hearing disabilities (hearing aids) because hearing impairment is relatively common in older people [19] and the ATs available for hearing disabilities are perhaps more familiar to these participants. It is possible that someone who is familiar with AT for one disability would be more amenable to using other AT including that available for dementia sufferers. The focus group interviews were independently transcribed and thematic analysis was used to determine the topics for inclusion in the questionnaire.

The questionnaire was constructed using the focus group data together with available evidence from literature. The questionnaire was divided into three sections. Section 1 asked the respondent (carer) four questions covering gender, age range, the amount of contact with the person they care for and their relationship. Section 2 included six questions covering some information about the person they were caring for. This section covered details about the dementia sufferer, their age and when the memory problem was diagnosed. The severity of the dementia sufferers' symptoms was gauged by asking how long the person could be left alone for (if at all) and by whether the person was living in the community or in residential/nursing care. Whether or not the person with dementia used ATD was also queried in this section with a space to list any devices used. The last question in this section focussed on problems affecting the person suffering from dementia and their carer. Five different problems that were mentioned by the focus groups and in literature were chosen. These were wandering or getting lost outside the home, losing things, finding activities to occupy free time, toileting/continence and eye/hearing tests. Although there is little evidence in the literature that hearing or eye tests might be a problem, the question was included because the NICE-SCIE guideline on supporting people suffering from dementia and their carers in health and social care [20] states that regular eye checks should not be forgotten. The third section of the questionnaire was titled "Some questions about memory problems and ways of coping" and was planned to encourage respondents to focus on needs and on how the needs were being met. The first question in this section listed six "Carer concerns", all of which had been brought up in focus group discussions. The other questions in this section covered unmet needs, any ATD that might be in use, and where people would look for information on ATD. The final question covered ATD that the carer might want to obtain, but did not have currently. Question 10 (covering common problems for people with dementia in Section 2) and question 11 (covering common concerns for dementia carers in Section 3) asked for a three point rating scale response to each of the problems/concerns listed. This could be either "not a problem", "Sometimes difficult/or slight problem" or "Often difficult/or big problem". The questionnaire was distributed at the three 'South Buckinghamshire' groups that had not taken part in the focus group work and at both the 'Templeton café groups. After a brief introduction to the project, participants were offered the choice of filling in the questionnaire during the group meeting or taking it home to complete later. A stamped addressed envelope was offered to the few people who elected to complete the document later. It would have been insensitive to exclude bereaved carers or those who contacted their loved one less than once per week, so everyone who wanted to complete the questionnaire was allowed to do so, however bereaved carers and those who had less than weekly contact were excluded from the subsequent analysis.

\subsection{Participants}

All the participants in this study were informal carers of people with dementia who belonged to Alzheimer's society carer support groups. Carer views were elicited rather than those of dementia sufferers themselves for two main reasons. Firstly, many of the ATD interventions provide reassurance for carers rather than provide help that would be immediately perceived as beneficial by the dementia sufferer. For example a wander alarm may be unnoticed or perceived as annoying by a dementia sufferer, but be reassuring and helpful for the carer. Secondly, problems with memory and communication are classic symptoms of dementia and these problems could make it difficult for dementia sufferers to accurately report ATD use. In addition, family and friends are likely to be instrumental in sourcing and implementing the use of ATD.

Focus group participants were all current or former carers of people with dementia, who were able to give informed consent to take part in the group. Although no formal personal information was taken, all members of both focus groups appeared to be well over the age 
of 65. One of the groups had two men and four women taking part, the other was composed of one man and five women.

\subsubsection{Questionnaire respondents}

50 carers completed the questionnaire but for eight of these respondents the care-recipient was deceased. The responses from bereaved carers were not included in the analysis because the questionnaire was worded in the present so it was difficult for people to fill in accurately for past events. For example "what is their age now?" caused confusion; with some people calculating how old their loved one would have been had they not died, while others filled in the person's age at death. There was also a potential for carers to have forgotten the details of events, particularly as in some cases the person with dementia had passed away years previously (six years in one case). Of the 42 remaining respondents, 12 men and 30 women, 24 were over 70 years old and only 2 were under 50. Eight carers were children of the person they cared for and the remaining 34 were spouses (32) or partners (2). $62 \%$ (26) of carers lived with the person they cared for, with another $14 \%$ (6) visiting or phoning every day. The remaining $24 \%$ (10) had at least weekly contact with the person they cared for.

\subsubsection{Characteristics of Dementia suffers being cared for by respondents}

The ages of dementia sufferers reported on in this study ranged from 56 years to 92 years with an average age of 76.5 years. The mean age at diagnosis was 71.9 (range 54 years -90 years old) with $40 \%$ of the total sample being less than 70 when the disease was diagnosed. The number of years since diagnosis ranged between 1year and 13 years (Mean 4.5 years) with 22 diagnoses between one and four years ago, 15 between five and eight years ago and 5 between nine and thirteen years ago. One respondent chose not to answer the 'time since diagnosis question and another marked the question "unknown". Almost half the sufferers (19) were judged to be unsafe to be left alone, thirteen could be left for short periods (up to 2 hours) and eight could be left all day or overnight. Fourteen dementia sufferers $(33 \%)$ were living in residential or nursing homes at the time of the survey.

\section{Results}

\subsection{Focus group results}

The focus group results provided interesting information beyond what was needed to develop the questionnaire. It was particularly interesting to note that no one in the either of the groups had personal experience of using any ATD with the person they care for, or in their immediate circle. Although some of the group members eventually remembered having heard about ATD, it was clear that few people had considered the possibility that this type of technology would be useful to them.

In an attempt to be supportive, the Alzheimer's society area manager gave out the society's Assistive technology information brochure at the meeting one month prior to the data collection. Although most focus group members had received this information, only one group member (in Group 2) actually remembered receiving the information. It seems unlikely that this information would have had much influence on this study because so little of it was recalled by the group members. Interestingly, although group 1 were initially adamant that they didn't know of any ATD and had not received any information on it, as the session progressed participants began to remember some devices. Group 1 mentioned movement sensors "there is technology already isn't there for when people start moving around at night that goes to a call centre." and wander alarms "But isn't there a device to warn the carer wherever they are?" In Group 2, where one participant remembered being given information on ATD the previous month, nobody mentioned knowing about any devices. Group 2 did remember AT advertised in magazines and papers but mentioned that it was all for physical problems and in that any case it was difficult to know whether any of the advertised devices were actually any good. It was generally agreed in group 2 that the Alzheimer's society area manager was the best source of information along with other group members. Social services were felt to be ill informed compared with the Alzheimer's society group. A question about where people would ask for information was included in the questionnaire in order to find out where the information about the latest developments could be most usefully targeted. Group 2 seemed to be more negative toward the concept of ATD making comments like "I don't think there is anything really.", "What is there? What can they do?" and "But you see the thing is we're the carers and we don't need that and they're too far gone to use 
that. We don't need those things really do we, well we don't because we're the carers." Whereas group 1 had already begun talking about possible ATDs before the session had officially started "Couldn't there be a sort of mechanical thing, because of all this modern equipment, couldn't they have something round their neck with some sort of recording in reminding them on a daily basis, something like that. Would that be possible do you think?"

\subsubsection{Common problems}

A number of problems and concerns were mentioned by both focus groups. In particular both groups mentioned that they cared for people who could not be left alone. Safety both inside the house, particularly in the kitchen and outside the house were major areas of concern for both groups. Both groups were concerned about people leaving the home and getting into difficulties. Strategies to reduce risks included locking doors and labelling clothes with name and address. Both groups reported problems with losing things, in some instances these were things that carers had hidden to prevent the person with dementia from accessing them; for example one participant said "But that's what your life is like isn't it, you're constantly hiding things. . . I had so many things hidden in the tops of cupboards and wardrobes and I'm still, I find I still. . . certain things have never been unearthed again but when you want to find anything you forget where they are." Both groups of carers indicated that they found the behaviour of the person they cared-for frustrating at times. For example "You can't please him at all." And that frustration became distress when talking of managing continence problems "Because it almost seems like torture to change them sometimes." Continence was reported as a problem by both groups.

A question was asked about hearing aid use in order to gauge the level of familiarity the group members had with well established AT. With both groups this provoked some discussion about hearing and sight tests and the difficulties of knowing whether or not the person with dementia could actually see or hear.

\subsubsection{Ideas for potential Assistive Technology for people with Dementia (ATD)}

The focus group members suggested a number of ideas for ATDs but most of these were devices that already exist; indicating that lack of awareness of what is available may be major factor in the lack of ATD use. (Table 1 shows the descriptions of suggested devices and corresponding commercially available product).
Two other potential devices were suggested. One suggestion "I wonder if there is any way you can bring music or whatever into some things which would remind them." This type of device exists as a research prototype but is not yet available commercially. The Computer Interactive Reminiscence and Conversation Aid (CIRCA) project [13] developed an interactive multimedia computing system that allowed dementia patients to choose music, pictures and short films to hear and see, the device successfully promoted reminiscence and communication. The CIRCA device would probably fulfil this requirement. The other need mentioned in the context of possible ATD was "The only thing that I need at the moment is somebody who could interpret my husband's speech because I can't understand him, half the time I can't understand him.” Although simple low-tech visual communication aids are available (eg The Visual Book for the Care Sector by Visual Communication Aids Ltd.) there is as yet, no device to clarify mumbled speech or correct wording where this is confused.

\subsection{Questionnaire results}

\subsubsection{Carer concerns}

Table 2 presents responses to the question "The following concerns often worry carers of people with memory problems. Please mark how concerned you are about the following:" The carers' most frequently highlighted concern was "getting frustrated with the person you care for" with 74\% (31) respondents reporting the problem, 20 of whom marked frustration as often difficult or a big problem. Four carers chose not to respond to this question. The carers second most frequent concern was "Understanding what the person you care for wants" which was reported by 64\% (27) carers, of whom 11 marked understanding as a major problem. Five people chose not to respond to this question. Fewer carers reported worries about safety in the home (21) and eating (22). "Safety outside the home" and "safety in the kitchen" were problematic for $48 \%$ (20) of carers surveyed. The non response rate to these questions was relatively high with 12 non-responses for outdoor safety and 10 for kitchen safety. This is probably because where people had been placed in residential care, these issues were no longer a concern for the carer, but they did not want to imply that their loved one was safe outside or in the kitchen. 
Table 1

Device suggested by focus groups that already exist

\begin{tabular}{|c|c|c|}
\hline $\begin{array}{l}\text { Focus } \\
\text { group }\end{array}$ & Description of potential AT & Existing device \\
\hline 1 & $\begin{array}{l}\text { if I could only sort of keep telling her this for the whole morning perhaps it will help. So could it } \\
\text { be possible to get some device like one of these where I could record on it, she could put it round } \\
\text { her neck and every, say, } 20 \text { minutes, remind her }\end{array}$ & $\begin{array}{l}\text { Memo - minder } \\
\text { such as VoiceCue } \\
\text { or Minder }\end{array}$ \\
\hline 1 & So if there was only something I could record and it kept on. & proximity \\
\hline 2 & $\begin{array}{l}\text { if a voice came to her and said, "Margaret it's time for your lunch now. Go and get your sandwiches } \\
\text { out of the fridge," she might do that and she might have done it and. . }\end{array}$ & voice memo \\
\hline 1 & $\begin{array}{l}\text { The other thing you could think about is what you do to criminals put a tag. .. } \\
\text { An electronic tag. }\end{array}$ & $\begin{array}{l}\text { personal locator } \\
\text { device such as } \\
\text { Care Trak or } \\
\text { ilocate }\end{array}$ \\
\hline 1 & $\begin{array}{l}\text { Now if there could be a little device, something like that which you could programme, sort of, } \\
\text { spectacles, dentures, you can't find them-just press it and it tells you where it is. Yes a buzz comes } \\
\text { from somewhere. }\end{array}$ & $\begin{array}{l}\text { Locator device } \\
\text { such as Loc8tor } \\
\text { pack or }\end{array}$ \\
\hline 1 & So if something could be devised and you could programme it on all the various things & Smartfinder \\
\hline 2 & $\begin{array}{l}\text { I was looking all over the house for it and couldn't find it and it would have been useful to have } \\
\text { a device that says, "The watch is on top of the cupboard," but in the end I found it on top of the } \\
\text { cupboard. }\end{array}$ & locator \\
\hline 2 & ... if you had something of a clock that could talk to the person and say their name... & $\begin{array}{l}\text { Speaking clock } \\
\text { Talking LCD } \\
\text { alarm clock }\end{array}$ \\
\hline
\end{tabular}

Table 2

Level of carer concern about selected issues

\begin{tabular}{lccrc}
\hline Concerns of carers & $\begin{array}{c}\text { Often difficult } \\
\text { or big problem }\end{array}$ & $\begin{array}{c}\text { Sometimes difficult } \\
\text { or slight problem }\end{array}$ & $\begin{array}{c}\text { No } \\
\text { problem }\end{array}$ & $\begin{array}{c}\text { No } \\
\text { response }\end{array}$ \\
\hline Getting frustrated with the person you care for & 20 & 11 & 6 & 5 \\
Understanding what the person you care for wants & 16 & 11 & 10 & 5 \\
Concerns about eating & 9 & 12 & 13 & 8 \\
Accidents in the kitchen & 7 & 13 & 12 & 10 \\
Safety at in the home & 5 & 17 & 11 & 9 \\
Safety outside the home & 5 & 15 & 10 & 12 \\
\hline
\end{tabular}

\subsubsection{Dementia sufferer problems as perceived by the} carer

Responding to the question "The following problems commonly affect people with memory problems. Please mark how much of a problem these are in your experience with your relative or friend" carers indicated that losing things was the most troubling symptom with $62 \%(n=26)$ reporting losing things as a "big problem/often difficult". Table 3 shows dementia sufferers' problems as perceived by the carer. The response rate to the question was low with 11 out of the 42 respondents failing to mark any box. Toileting/continence problems were the second most important difficulty, marked as a big problem by $24 \%$ of respondent with a further $43 \%$ indicating that continence was a slight or occasional difficulty. Finding activities to occupy free time and Eye/hearing tests were also marked as big problems by $24 \%$ of respondents. The least frequently reported problem was wandering/getting lost, but this was still a significant problem with $48 \%$ of carers reporting at least some difficulties in this area.

\subsubsection{Assistive Technology and unmet needs}

$95 \%$ of respondents indicated that the person they cared for did not use any ATD and the remaining two respondents left the question blank. When asked "Do you know of any technology for people with memory problems, that the person you care for doesn't have, that you think would be useful?" $93 \%$ indicated that they did not know of any potentially useful ATD. One person marked the yes box but listed a walking frame as the AT that was needed. Another ticked the yes box but the device listed was undecipherable and a third failed to answer the question. Reporting on the use of other AT (not for dementia) by the person they cared for $36 \%$ of respondents indicated that the person did use some form of AT. The most common AT use reported was a wheelchair used by 10 out of the 15 people who used any AT. Four carers reported that their relative had a 
Table 3

Problems of dementia sufferers as perceived by their carers

\begin{tabular}{lccrc}
\hline Problems & $\begin{array}{c}\text { Often difficult } \\
\text { or big problem }\end{array}$ & $\begin{array}{c}\text { Sometimes difficult } \\
\text { or slight problem }\end{array}$ & $\begin{array}{c}\text { No } \\
\text { problem }\end{array}$ & $\begin{array}{c}\text { No } \\
\text { response }\end{array}$ \\
\hline Losing things & 26 & 7 & 5 & 4 \\
Toileting/continence problems & 10 & 18 & 11 & 3 \\
Finding activities to occupy free time & 10 & 11 & 3 & 8 \\
Eye/hearing tests(at hospital or optician) & 10 & 13 & 14 & 5 \\
Wandering/getting lost outside the home & 9 & 11 & 11 & 11 \\
\hline
\end{tabular}

hearing aid but two of these said the person would not actually use the aid. Three people reported the use of a walking frame with two of these also using a walking stick. Only one person listed glasses as an AT in use although it seems likely that many more people did use glasses but did not consider them to be AT.

Most carers did not feel that the person they cared for had unmet needs but $29 \%$ of carers (12) expressed the view that some needs were not being met. Nine of these 12 carers listed the unmet needs on the questionnaire; the most frequently mentioned needs were for leisure/ social activities which were listed by three carers. Two other carers mentioned needs that may also be related to social and leisure needs 1) a shortage of staff at the home 2) an extra day at the day centre. Two carers mentioned AT needs; one felt their relative needed help with mobility and another wanted a wheelchair. One carer wanted home physiotherapy, hydrotherapy and help with finances. One carer, who recorded that their relative had been diagnosed with dementia three years ago, mentioned the need for proper investigation and diagnosis of dementia. Living arrangements did not appear to have a huge impact on carers' perception of unmet needs, with seven out of 27 (26\%) carers of community dwelling dementia sufferers reporting unmet need and five out of 14 (36\%) of carers whose loved ones lived in institutions reporting that needs were not fully met.

\subsubsection{Information seeking}

In response to the question "If you wanted information on technology for memory problems, where would you ask for advice? (Please tick all that apply)" most carers $(67 \%)$ said they would ask for information on ATD from the Alzheimer's Society with the GP being the next most popular source of information. Only one carer ticked the 'other' option where he listed the Admiral Nurse, specialist mental health nurses promoted and developed by Dementia ${ }^{\mathrm{UK}}$, for his preferred source of information. (Table 4 shows the where carers would ask for information on ATD if they wanted to learn more). Hospital clinics or consultants would
Table 4

\begin{tabular}{lc}
\multicolumn{2}{c}{$\begin{array}{c}\text { Table } 4 \\
\text { Preferred information source for ATD }\end{array}$} \\
\hline Information source & $\begin{array}{c}\text { Number of respondents } \\
\text { choosing to ask }\end{array}$ \\
\hline Alzheimer's society & 28 \\
GP/Family Doctor & 16 \\
Hospital/Consultant & 13 \\
Social services & 10 \\
Occupational Therapist & 9 \\
Internet & 9 \\
Friends & 5 \\
Library & 1 \\
Other (Admiral nurse) & 1 \\
Disabled Living Foundation & 0 \\
\hline
\end{tabular}

Total number of responses 92.

be approached by $31 \%$ of relatives and Social services would be approached by $24 \%$ of carers looking for ATD information. Occupational therapists and the internet were equally popular resources with $21 \%$ of carers consulting each of these information sources. Friends and relatives were a source of information for only $12 \%$ of respondents. Just one carer indicated the library as a source of information. The Disabled Living Foundation is a UK charity whose purpose is to provide impartial advice, information and training on daily living aids but none of the carers considered this organisation as a source of information on ATD.

\section{Discussion}

\subsection{Study limitations}

The main limitations of this study were the small sample number and the restricted geographical area in which the study took place; just two adjacent administrative areas were included in the project. It is possible that in other areas in the country, local councils may have a more proactive attitude towards assistive technology so that staff and subsequently service users would be better informed about ATD. Another limitation of this study was the number of missing responses, particularly for the two questions where concerns and problems were listed. This could be an indication 
that unlisted problems were the main concerns for these carers, however, those respondents with many missing answers also failed to list any unmet needs. It is possible that filling the questionnaires in during the carer support meeting was not optimal for some carers, leaving them either too rushed or distracted to complete the questions that required more thought.

\subsection{Potential for ATD use}

The focus group discussion brought out a great deal of information on needs that were not being met, many of which could potentially be satisfied by the use of commercially available ATD. The survey elicited many responses to listed problems and areas of concern but most of the respondents did not consider these to be unmet needs in the following question on the survey. Clearly some of the problems and concerns which the carers reported on in the questionnaire could be classed as unmet needs because if issues were being adequately dealt with, far more carers would have reported no problems or concerns. In fact only one carer out of 42 rated all eleven problems and concerns listed in the survey as being 'No problem'. From the six carer concerns, at least three (Accidents in the kitchen Safety in the home, Safety outside the home,) could be alleviated by commercially available ATD. Communication books for older people are also available [22] that could help with "Understanding what the person you care for wants" which could in turn, reduce carer frustration. There are AT solutions for feeding and eating, but these would not necessarily alleviate the "Concerns about eating" that can be associated with dementia, when people may over or under eat, or consume inappropriate substances. Similarly ATD solutions exist to help people manage with "Wandering/getting lost outside the home" and "losing things". "Toileting/continence problems" are difficult to address with ATD but clearly marked toilets and additional lighting are thought to help maintain continence. "Finding activities to occupy free time" was marked as a problem by $50 \%$ of questionnaire respondents and where people had taken time to fill in unmet needs in the open section of the question this problem was mentioned most often. This theme was also evident in both focus groups. The loss of social skills and reading ability and problems with executive thinking mean that many of the pastimes and entertainments that people enjoyed in previous years are no longer enjoyable or even possible for them. There is a clear need for some sort of entertainment system or aid to interaction, to facilitate conversation. This need has been addressed to some extent by the work of the CIRCA project [13] but this technology is not yet commercially available.

\subsection{Lack of ATD use}

In the carer sample studied in the Focus group work $(n=12)$ and the survey $(n=42)$, none of the carers had any personal experience of ATD use. Although this was a very small sample compared with the number of dementia sufferers in the UK, the lack of ATD use shown by this study was an indication that ATD was under used by dementia sufferers and their carers. Since none of the participants had any first hand experience of ATD use, it was difficult to obtain direct information on barriers to ATD use from this study. Lack of funding for desired ATD does not seem to have been a barrier to up take for this group of carers because none of them reported not having ATD that might be useful in their situation. Lack of awareness is a possible barrier, but a number of the focus group participants had been given a leaflet on ATD the month before the focus group study and during the group discussion some people did remember hearing about specific ATD. Clearly, some focus group participants did have a limited awareness of ATD. However, when asked to talk about potential technological developments to cope with the day to day problems of dementia, sufferers and carers described situations with technologies that already exist commercially. Some focus group participants were enthusiastic about the potential benefits of ATD while others seemed to feel nothing could be done to help them, or the person suffering from dementia. There is a view that older people are generally reluctant to engage with newer technologies, which may discourage carers from investigating ATD. However in this study more than $20 \%$ of carers said they would look for information on the internet indicating that in the future, with more people being accustomed to using computers, fear of technology is likely to be a decreasing barrier to ATD uptake. National Statistics from quarter one 2011 [21] states that $76 \%$ of the $75+$ age group had never used the internet, but in the 65 to 74 age group, $57 \%$ of UK citizens were internet users. Since the data collection, more information about commercially available ATD has been available on the internet, for example the AT Dementia site [23] went live in 2007, which provides information on both Telecare as well as stand alone products. The Alzheimer's society launched their own a web based shop in June 2011 [24] to help carers to obtain useful ATD products. 
Lack of information about appropriate ATD is undoubtedly a factor in non-use and a potential barrier to ATD uptake. Carers are undoubtedly under a great deal of strain [6] and this burden is not always relieved even when the person they are supporting enters residential care [25]. It could be that the burden of care leaves the caregiver without the time or emotional energy to investigate technological solutions to the difficulties they are faced with. Since most carers in this study would look to the Alzheimer's society to provide them with information, the society's recent launch (2011) of an online shop for these products promises to be very helpful. Survey results indicate that primary and secondary care physicians need to be made aware of the latest developments in ATD. It is interesting that no one would consider the Disabled Living Foundation as a source of information about ATD. This could indicate a rejection of the "disabled" status and the stigma involved, or that the carers didn't consider dementia to be a disability.

\section{Conclusions}

This study showed that there was a lack of awareness of ATD, among family carers, and its potential to alleviate carer burden and promote independence for dementia sufferers. Although commercially available ATD products already exist to alleviate many difficulties that dementia/carers dyads experience, appropriate and timely information is needed to enable people to benefit from these devices. The areas of leisure, entertainment and social interaction are not well served by current commercially available ATD, yet these activities are vital for maintaining quality of life, so development of new ATD in these areas would be welcome. Similarly, continence and toileting is a fundamentally important field where a discreet technology that maintains continence might be usefully developed. Further work is also necessary to investigate ATD use in the community, to provide an evidence base for ATD use and to identify where products need to be improved.

\section{Acknowledgments}

The Authors would like to thank Professor Sheila Kitchen and Dr Ruth Mayagoitia-Hill of King's College, London for their support and advice with this work, the Alzheimer's society (South Buckinghamshire and Harrow and Hillingdon) for facilitating the data collection and all the carers who took the trouble to share their experiences.

\section{References}

[1] J. Wanacata, M. Musalek, R. Alexandrowicz and M. Krautgartner, Number of dementia sufferers in Europe between the years 2000 and 2050, European Psychiatry 18 (2003), 306313.

[2] Audit Commission (2004) Support for Carers of Older People, Audit Commission, London. http://www.audit-commission. gov.uk/nationalstudies/health/socialcare/pages/olderpeople5. aspx\#downloads.

[3] R. Schulz and L. Martire, Family Caregiving of Persons With Dementia: Prevalence, Health Effects, and Support Strategies, American Journal of Geriatric Psychiatry 12 (2004), 240-249.

[4] M. Ory, R. Hoffman III, J. Yee, S. Tennstedt and R. Schulz, Prevalence and Impact of Caregiving: A Detailed Comparison Between Dementia and Nondementia Caregivers, Gerontologist 39 (1999) 177-185.

[5] R. Schulz and S.R. Beach, Caregiving as a risk factor for mortality: The Caregiver Health Effects Study, Journal of the American Medical Association 282 (1999), 2215-2260.

[6] J. Georges, S. Jansen, J. Jackson, A. Meyrieux, A. Sadowska and M. Selmes, Alzheimer's disease in real life-the dementia carer's survey, International Journal of Geriatric Psychiatry 23 (2008) 5, 546-551.

[7] C. McCreadie and A. Tinker, The acceptability of assistive technology to older people, Ageing and Society 25 (2005), 91-110.

[8] H. Hoenig, D. Taylor and F. Sloan, Does assistive technology substitute for personal assistance among the disabled elderly? American Journal of Public Health 93 (2003), 330-337.

[9] P. Duff and C. Dolphin, Cost-benefit analysis of assistive technology to support independence for people with dementia Part 2: results from employing the ENABLE cost-benefit model in practice, Technology and Disability 19 (2007), 79-90.

[10] J. Barlow, S. Bayer and R. Curry, Flexible Homes, Flexible Care, Inflexible Organisations? The Role of Telecare in Supporting Independence, Housing Studies 20 (2005), 441-456.

[11] A. Bowes and G. McColgan, Implementing telecare for people with dementia: supporting ageing in place in West Lothian, Scotland, Journal of Care Services management 3 (2009), 227-243.

[12] J. Hoey, A. V. Bertoldi, T. Craig, P. Poupart and A. Mihailidis, Automated handwashing assistance for persons with dementia using video and a partially observable markov decision process, Computer Vision and Image Understanding 114 (2010), 503-519.

[13] G. Gowans, J. Campbell, N. Alm, R. Dye, A. Astell and M. Ellis, Designing a multimedia conversation aid for reminiscence therapy in dementia care environments, Extended Abstracts on Human Factors in Computing Systems (2004) 825-836 published by ACM Press New York.

[14] N. Alm, A. Astell, M. Ellis, R. Dye, G. Gowans and J. Campbell, A cognitive prosthesis and communication support for people with dementia, Neuropsychological Rehabilitation 14 (2004), 117-134.

[15] E.F. LoPresti, A. Mihailidis and N. Kirsch, Assistive technology for cognitive rehabilitation: state of the art, Neuropsychological Rehabilitation 14 (2004), 5-39.

[16] A. Mihailidis, J. Barbenel and G. Fernie, The efficacy of an intelligent cognitive orthosis to facilitate handwashing by persons with moderate to severe dementia, Neuropsychological Rehabilitation 14 (2004), 135-171.

[17] I. Mansell, G. Bennett, R. Northway, D. Mead and L. Moseley, The learning curve: the advantages and disadvantages in the 
use of focus groups as a method of data collection, Nurse Researcher 11 (2004), 79-88.

[18] D. Morgan, Focus Groups Annual Review of Sociology 22 (1996), 129-152.

[19] B.B. Abutan, A.W. Hoes, C.L. Van Dalsen, J. Verschuure and A. Prins, Prevalence of hearing impairment and hearing complaints in older adults: a study in general practice, Family Practitioner 10 (1993), 391-395.

[20] NICE-SCIE Guideline on supporting people with dementia and their carers in health and social care. National Clinical Practice Guideline Number 42 (2007) published by The British Psychological Society and Gaskell.
[21] http://www.visualcommunicationaid.com/products_csbook. html.

[22] Office for National Statistics. Internet Access Quarterly Update $18^{\text {th }}$ May, (2011) Edited by M. Williams retrieved from http://www.statistics.gov.uk/articles/nojournal/internetaccess-q1-2011.pdf.

[23] http://www.atdementia.org.uk/productSearch.asp?page_id= 16.

[24] http://alzheimers.org.uk/shop.html.

[25] M. Stephens, P. Ogrocki and J. Kinney, Sources of stress for family caregivers to institutionalized dementia patients. Journal of Applied Gerontology 3 (1991) 328-42. 